This item was submitted to Loughborough's Research Repository by the author.

Items in Figshare are protected by copyright, with all rights reserved, unless otherwise indicated.

\title{
Acute ibuprofen ingestion does not attenuate fatigue during maximal intermittent knee extensor or all-out cycling exercise
}

PLEASE CITE THE PUBLISHED VERSION

https://doi.org/10.1139/apnm-2018-0432

\section{PUBLISHER}

NRC Research Press (C) The authors

VERSION

AM (Accepted Manuscript)

\section{PUBLISHER STATEMENT}

This work is made available according to the conditions of the Creative Commons Attribution-NonCommercialNoDerivatives 4.0 International (CC BY-NC-ND 4.0) licence. Full details of this licence are available at: https://creativecommons.org/licenses/by-nc-nd/4.0/

\section{LICENCE}

CC BY-NC-ND 4.0

\section{REPOSITORY RECORD}

Morgan, Paul T., Anni Vanhatalo, Joanna L. Bowtell, Andrew M. Jones, and Stephen Bailey. 2019. "Acute Ibuprofen Ingestion Does Not Attenuate Fatigue During Maximal Intermittent Knee Extensor or All-out Cycling Exercise". figshare. https://hdl.handle.net/2134/34347. 


\section{Acute ibuprofen ingestion does not attenuate fatigue during maximal intermittent knee extensor or all-out cycling exercise}

Original investigation

Paul T. Morgan, Anni Vanhatalo, Joanna L. Bowtell, Andrew M. Jones and Stephen J. Bailey ${ }^{1}$

Department of Sport and Health Sciences, College of Life and Environmental Sciences, University of Exeter, St. Luke’s Campus, Heavitree Road, Exeter, EX1 2LU, UK.

\section{Address for Correspondence:}

Anni Vanhatalo, Ph.D.

Department of Sport and Health Sciences, College of Life and Environmental Sciences, University of Exeter, St. Luke’s Campus, Heavitree Road, Exeter, EX1 2LU, UK.

Tel: 01392722815

E-mail1: A.Vanhatalo@exeter.ac.uk

E-mail²: P.T.Morgan@exeter.ac.uk

E-mail ${ }^{3}$ : J.Bowtell@exeter.ac.uk

E-mail4: A.M.Jones@exeter.ac.uk

E-mail ${ }^{5}$ : S.Bailey2@lboro.ac.uk

${ }^{1}$ Present address for Stephen $\mathbf{J}$ Bailey: School of Sport, Exercise and Health Sciences, Loughborough University, Ashby Road, Loughborough, Leicestershire LE11 3TU

Running title: Influence of ibuprofen on skeletal muscle fatigue

Abstract word count: 250 words

Text-only word count: 5031 words

Disclosure of funding: This research was not supported by any funding body external to University of Exeter 


\section{ABSTRACT}

2 Purpose: Recent research suggests that acute consumption of pharmacological analgesics can improve exercise performance, but the ergogenic potential of ibuprofen (IBP) administration is poorly understood. This study tested the hypothesis that IBP administration would enhance maximal exercise performance. Methods: In one study, 13 physically active males completed $60 \times 3$-s maximum voluntary contractions $(\mathrm{MVC})$ of the knee extensors interspersed with a 2-s passive recovery period, on two occasions, with the critical torque (CT) estimated as the mean torque over the last 12 contractions (part A). In another study, 16 active males completed two 3-min all-out tests against a fixed resistance on an electrically-braked cycle ergometer with the critical power (CP) estimated from the mean power output over the final 30-s of the test (part B). All tests were completed 60 min after ingesting maltodextrin (placebo, PL) or $400 \mathrm{mg}$ of IBP. Peripheral nerve stimulation was administered at regular intervals and electromyography was measured throughout. Results: For part A, mean torque (IBP: $60 \pm 12$ vs. PL: $58 \pm 14 \%$ of pre-exercise MVC) and CT (IBP: $40 \pm 15$ vs. PL: $41 \pm 16 \%$ of pre-exercise MVC) were not different between conditions $(P>0.05)$. For part $B$, end-test power output (IBP: $292 \pm 28 \mathrm{~W}$ vs PL: $288 \pm 31 \mathrm{~W}$ ) and work done (IBP: $65.9 \pm 5.9 \mathrm{~kJ}$ vs PL: $65.4 \pm 6.4 \mathrm{~kJ}$ ) during the 3 -min all-out cycling tests were not different between conditions (all $P>0.05)$. For both studies, neuromuscular fatigue declined at a similar rate in both conditions $(P>0.05)$. Conclusion: Acute ingestion of $400 \mathrm{mg}$ IBP does not improve single-leg or maximal cycling performance in healthy humans.

Key words: Electromyography; neuromuscular fatigue; non-steroidal anti-inflammatory drugs; single leg exercise; whole-body cycling exercise 
Ibuprofen (IBP), a non-steroidal anti-inflammatory drug (NSAID) predominantly used to treat pain and reduce inflammation and fever, is considered safe for oral ingestion within healthy populations at the standard therapeutic dose (Albert \& Gernaat, 1984; García-Martín et al. 2004). Consequently, utilisation of analgesic (i.e., pain relieving) and anti-inflammatory medication, such as IBP, has emerged as a popular pre-competition strategy in elite athletes attempting to enhance athletic performance (Alaranta et al. 2008; Corrigan \& Kazlauskas, 2003; Da Silva et al. 2015; Gorski et al. 2011; Huang et al. 2006). It is believed that NSAIDs act centrally and peripherally to reduce the perception of pain and tissue inflammation, respectively (Friden \& Lieber, 1992). The therapeutic effect of NSAIDs in treating inflammation and pain has largely been ascribed to inhibited synthesis of prostaglandins (i.e. $\mathrm{PGE}_{2}$, Friden \& Lieber, 1992). Specifically, IBP limits the metabolism of arachidonic acid, a precursor for the synthesis of prostaglandins, by inhibiting the enzyme, cyclooxygenase (Albert \& Gernaat, 1984).

Following the onset of muscle contractions, changes in contraction-induced mechanical stimuli and noxious chemicals (including an increased $\mathrm{PGE}_{2}$ release) activate and/or sensitise molecular receptors located on the terminal end of group III and IV nerve fibers, contributing to an increased sensation of muscle pain during exercise (O’Connor \& Cook, 1999; McCord \& Kaufmann, 2010; Pollak et al. 2014). The activation of these receptors appears to play a role in neuromuscular fatigue development through modulating both central and peripheral fatigue. Indeed, when the ascending projection of group III and IV muscle afferents is attenuated via intrathecal fentanyl administration, central motor drive is increased (as inferred via electromyography, EMG) and peripheral fatigue development is expedited (Amann et al. 2009, 2011; Blain et al. 2016). Therefore, it is possible that an intervention that is able to 
reduce the magnitude of afferent feedback, such as NSAID administration, may attenuate the decline in skeletal muscle activation during intense exercise and thus improve exercise performance (Amann \& Calbet, 2008; Morgan et al. 2018a; Morgan et al. 2018b). Indeed, elevating the magnitude of muscle afferent feedback is known to impair endurance exercise capacity (i.e. Amann et al. 2013).

Acute consumption of analgesics (e.g. acetaminophen, ACT) has been shown to improve exercise performance (Foster et al. 2014; Mauger et al. 2010; Morgan et al 2018a; Morgan et al. 2018b). Mauger et al. (2010) administered an acute dose of 1.5 g ACT to trained cyclists and reported a 2\% improvement in 16.1-km time-trial (TT) performance. Moreover, an acute dose of ACT has been shown to improve exercise tolerance in the heat (Mauger et al. 2014), repeated sprint performance (Foster et al. 2014), repeated maximal voluntary contraction (MVC) performance of the knee extensors (Morgan et al. 2018a) and maximal cycling performance (Morgan et al. 2018b). However, while these studies suggest that oral administration of a commercially-available pharmaceutical analgesic can delay fatigue development and improve exercise performance (Foster et al. 2014; Mauger et al. 2010; Morgan et al 2018a, b), the ergogenic effects of IBP are unclear despite widespread use of IBP as a putative performance aid (Cleak, \& Eston. 1992; Nosaka \& Clarkson, 1996).

In contrast to the effects of ACT consumption on exercise performance, there is currently very limited published research on NSAID and NSAID-like compounds during exercise when muscle damage is not present. Despite the prevalence of its use amongst athletic populations, it is unclear whether IBP will provide an ergogenic benefit to performance in a ‘fresh' state. Other NSAID-like compounds have elicited no ergogenic effect on endurance performance (e.g., aspirin, Cook et al. 1997; ginger, Black \& O’Connor, 2008). In contrast, 
enhanced aerobic exercise performance following caffeine ingestion has been related, at least in part, to altered pain perception and enhanced endurance performance (e.g., Gonclach et al. 2016). In addition, whilst exercise has been shown to increase $\mathrm{PGE}_{2}$ release (Trappe et al. 2001; Novak \& Wennmalm, 1979; Wilson \& Kapoor, 1993; Vinikka et al. 1984), the extent to which it plays an important role, especially following interventions aimed at reducing $\mathrm{PGE}_{2}$ synthesis, during endurance exercise in the absence of muscle damage is unclear.

The purpose of this study was to test the hypotheses that, compared to a placebo, acute consumption of $400 \mathrm{mg}$ IBP would increase total work done, and reduce the rate of fatigue development by enabling a better maintenance of muscle activation during exercise. Two studies are presented using two different types of exercise: a 5-min single-leg intermittent MVC test and a 3-min maximal whole body cycling test to provide a more comprehensive understanding of the ergogenic potential of IBP.

\section{MATERIALS AND METHODS}

Participants

Two independent groups of thirteen recreationally-active males (mean \pm SD: age $31 \pm 7$ years, height $1.76 \pm 0.08 \mathrm{~m}$, body mass $75 \pm 11 \mathrm{~kg}$ ), and sixteen recreationally-active males (mean \pm SD: age $29 \pm 9$ years, height $1.79 \pm 0.07 \mathrm{~m}$, body mass $77 \pm 8 \mathrm{~kg}$, $\dot{\mathrm{VO}}_{2 \text { peak }} 60.8 \pm 7.8$ $\mathrm{ml} \cdot \mathrm{kg}^{-1} \cdot \mathrm{min}^{-1}$ ), volunteered for the first (A) and second (B) part of this study, respectively. All participants provided written, informed consent to participate in this study, which was approved by the Sport and Health Sciences Ethics Committee at the University of Exeter. After being informed of the experimental procedures and associated risks, all participants completed a medical health questionnaire to ensure it was safe for them to consume IBP prior to performing exhaustive exercise, due to the potential contraindications associated with IBP 
ingestion. Participants were not consumers of any 'pain relief' or anti-inflammatory medication (prescription or non-prescription) over the course of the study. None of the participants had a history of motor or neurological disorders. Participants were instructed to arrive at the laboratory in a rested and fully hydrated state, at least $3 \mathrm{~h}$ post-prandial, and to avoid strenuous exercise and refrain from consuming caffeine and alcohol in the $24 \mathrm{~h}$ preceding each testing session. Participants were also instructed to consume their habitual diet and continue normal training activities for the experimental period. For part A and B, participants recorded their diet and physical activity for $7 \mathrm{~d}$ prior to the first experimental visit and then replicated this for all remaining experimental visits.

\section{Experimental Design}

Both protocols (part A and B) followed identical experimental designs to previous research conducted within our laboratory (Morgan et al. 2018a, 2018b). For part A (Morgan et al. 2018a), participants visited the laboratory on three occasions over a 3-4 week period with tests being conducted on an isokinetic dynamometer (Biodex System 3, Shirley, NY, USA). For part B (Morgan et al. 2018b), participants visited the laboratory on five occasions over a 5-6 week period and tests were conducted on an electronically braked cycle ergometer (Lode Excalibur Sport, Groningen, the Netherlands). Experimental tests were separated by at least 7, but no more than 9, days (part A) and for at least $72 \mathrm{~h}$ (part B), and were completed at a similar time of day ( \pm 90 mins). The first laboratory visits for each study were used to familiarise participants to the measurements and experimental protocols described below. Subsequently, participants performed the fatiguing protocol (s) under two conditions (see 'Experimental Protocol'): placebo (PL) and IBP.

\section{Experimental Protocol A (60 MVC protocol)}


The isokinetic dynamometer was initially adjusted so that the axis of rotation of the lever arm was in line with the lateral epicondyle of the right femur. Participants were seated with the hip and knee joints at relative angles of $155^{\circ}$ and $90^{\circ}$, respectively. The remainder of the chair settings were recorded (during familiarisation) and replicated in all subsequent tests to ensure identical body position throughout the experimental trials. Inelastic padded Velcro straps were fastened at the ankle, quadriceps, hip and shoulders to maintain a stable body position.

Following the familiarisation trial, visits 2 and 3 were completed in a double-blind, randomised fashion using a cross-over experimental design. $1000 \mathrm{mg}$ of maltodextrin (placebo) or $400 \mathrm{mg}$ IBP (combined with $600 \mathrm{mg}$ maltodextrin) was ingested orally, 60 minutes prior to the exercise bout such that the start of the exercise trial was expected to coincide with peak plasma [ibuprofen] concentration (Janssen \& Venema, 1985). For IBU, 2 identical capsules containing 200 mg ibuprofen and 300 mg maltodextrin each were ingested. The placebo was made from dextrose powder inserted into 2 gelatine capsules (500 mg in each capsule) designed to have a similar appearance and the weight to IBU capsules. The trials started with a standardised isometric warm-up routine (10 isometric contractions for $3 \mathrm{~s}$ at $50 \%$ of pre-exercise MVC as measured during familiarisation testing) and testing of the optimal EMG electrode, anode, and cathode placement and stimulation intensity for peripheral nerve stimulation. The experimental protocol consisted of 60 brief MVCs (3 s contraction, 2 s rest), in response to a visual prompt to 'go' and 'relax', accompanied by the same verbal instructions from the experimenter. Every $6^{\text {th }}$ contraction was accompanied by peripheral nerve stimulation during and $1 \mathrm{~s}$ post MVC (as described for pre-trial measurements below). Participants were not made aware of the time or the number of MVCs 
that had elapsed during the protocol and were instructed to continue to perform maximal contractions throughout.

\section{Experimental protocol B (3-min cycling test)}

For the 3-min cycling protocol, participants initially completed an incremental ramp test to exhaustion for the determination of gas exchange threshold (GET), linear factor, peak aerobic power output and peak oxygen uptake $\dot{\mathrm{V}}_{2 \text { peak }}$ as previously described (Black et al. 2014; Morgan et al. 2018b). The fixed resistance for the 3-min cycling protocol was set using the linear mode (i.e. linear factor) of the ergometer such that on reaching their preferred cadence, the participants would achieve a power output equivalent to $50 \%$ of the difference between GET and $\dot{\mathrm{VO}}_{2 \text { peak }}$ (linear factor $=50 \% \Delta$ peak aerobic power output $/$ preferred cadence $^{2}$ ). During this visit, the seat and handlebar positions were adjusted for comfort and replicated for all tests. During the second and third laboratory visits, participants completed a 3-min allout test with these serving as familiarization trials to the experimental protocol as described below and to ensure the coefficient of variation for work done and critical power (CP) between visits was $<1 \%$. Participants then performed the 3-min all-out cycling test under two conditions: placebo (PL) and IBP.

The experimental protocol consisted of a 3-min period of unloaded pedalling at each participant's preferred cadence (85-100 rpm), followed by a 3-min all-out sprint, 60 min following ingestion of either placebo (1000 mg maltodextrin) or $400 \mathrm{mg}$ of IBP. The order of trials on visits 4 and 5 were administered in a double-blind, randomised fashion using a crossover experimental design. The 3-min all-out cycling protocol used in this study replicated the procedures described previously by Vanhatalo et al. (2007, 2008). 
Neuromuscular function (Parts A and B)

For part A and B, neuromuscular function was assessed pre-, during- and post-trial (<10 s).

Single peripheral nerve stimulation pulses were manually triggered at rest to determine preexercise neuromuscular function, namely the characteristics of the M-wave response (Mwave amplitude; $\mathrm{M}_{\max }$ ) to supra-maximal nerve stimulation, voluntary activation (for part $\mathrm{A}$ only) and potentiated twitch torque (pTw, for part A only). During MVCs, peripheral nerve stimulation pulses were triggered to occur as soon as a peak torque was achieved (typically $1.5 \mathrm{~s}$ into a $3 \mathrm{~s}$ contraction) and were each separated by a $45 \mathrm{~s}$ rest period. The stimuli were also delivered 1-s after the cessation of the contraction to provide a resting pTw. Identical measurements were repeated as soon as possible $(<10 \mathrm{~s})$ after the fatiguing exercise to determine post-exercise neuromuscular function (see figure 1).

\section{Torque (Part A)}

For part A, knee-extensor torque from the Biodex isokinetic dynamometer was sampled at $1000 \mathrm{~Hz}$ and low-pass filtered at $40 \mathrm{~Hz}$, before being displayed on a wide screen monitor using Spike2 (CED, Cambridge, UK). Torque was expressed throughout as a percentage (\%) of initial pre-exercise MVC.

\section{Breath-by-breath pulmonary gas exchange (Part B)}

For the 3-min maximal cycling protocol, participants wore a face mask connected to an impeller turbine transducer assembly (Cortex Metalyzer, Cortex, Leipzig, Germany). Inspired and expired gas volume and concentration signals were continuously sampled at $100 \mathrm{~Hz}$. The analyser was calibrated before each test with gases of known concentration, and a calibration syringe of known volume (3-L; Hans Rudolph, KS). 
For parts $\mathrm{A}$ and $\mathrm{B}$, surface EMG activity was recorded from m.vastus lateralis, $m$.vastus medialis and $m$.rectus femoris of the quadriceps and $m$.biceps femoris of the hamstring of the right leg using active bipolar bar electrodes in a single differential configuration (DE2.1, DelSys Inc, Boston, MA, USA). The electrodes were placed over the respective muscle bellies (SENIAM guidelines). Double-sided adhesive tape and a hypoallergenic medical tape were used to ensure the EMG sensor stability. The skin area underneath each EMG electrode was shaved, then exfoliated and cleaned with alcohol to minimise the skin impedance. The EMG and torque signals were pre-amplified $(1,000 \mathrm{x})$, band-pass filtered $(20-450 \mathrm{~Hz}$,

Bagnoli-8, DelSys Inc, Boston, MA, USA), and then transferred to a computer with a sampling frequency of $2 \mathrm{kHz}$ and high-pass filtered at $10 \mathrm{~Hz}$. EMG and torque data were recorded continuously and digitised synchronously with 16 bit resolution via an A/D converter ( \pm 5 V range, CED 1401 power, Cambridge, UK). EMG was average rectified using the root mean square method (EMG $\mathrm{RMS}$ ). $\mathrm{EMG}_{\mathrm{RMS}}$ was then normalised to the pre-exercise maximum (or maximal EMG signal) and the local M-wave amplitude (closest time point measure of the M-wave) in order to exclude any changes to the EMG trace to changes in local excitability. The ground electrode was placed over the patella of the right leg.

\section{Peripheral Nerve Stimulation (Parts A and B)}

217 Electrical stimulation was applied with a constant current stimulator (Digitimer Stimulator 218 DS7A, Digitimer, UK) for the assessment of M-waves (parts A and B) and potentiated twitch force (part B). M-waves were elicited by supramaximal percutaneous electrical stimulation of the femoral nerve (200 $\mu$ s duration). The cathode was placed over the femoral nerve in the inguinal fossa, approximately $3-5 \mathrm{~cm}$ below the inguinal ligament in the femoral triangle. 
muscle action potential (i.e. M-wave) was monitored to identify the optimal position of the cathode for attaining maximal peak-to-peak M-wave amplitude. Once the M-wave was elicited, the maximum amplitude (peak-to-peak) of the $\mathrm{M}$-wave was determined $\left(\mathrm{M}_{\max }\right)$ for the vastus lateralis and vastus medialis. To determine the stimulation intensity, single stimuli were delivered in $20 \mathrm{~mA}$ step-wise increments from $100 \mathrm{~mA}$ until a plateau in quadriceps pTw (part A only) and M-wave were observed. To ensure a supramaximal response, the current was increased by an additional 30\% (mean \pm SD current $=214 \pm 66 \mathrm{~mA} ; 251 \pm 46$ $\mathrm{mA}$, part $\mathrm{A}$ and $\mathrm{B}$, respectively). The average $\mathrm{M}_{\max }$ was obtained from 3 stimuli, with $\sim 8-10 \mathrm{~s}$ separating each pulse at rest. For the 3-min maximal cycling protocol, single peripheral nerve stimulation pulses were manually triggered at 'rest' (defined as $80 \mathrm{rpm}$ at $20 \mathrm{~W}$ ) to determine pre-exercise neuromuscular function. Initially, the crank angle at which peripheral nerve stimulation was to be delivered during the trials was determined for each subject as described by Black et al. (2014) and as performed by Sidhu et al. (2012). Peripheral nerve stimulation pulses were triggered to coincide with maximal muscle activation around the crank cycle (typically around 50-60 from top-dead centre) 3 times, randomly, during a $10 \mathrm{~s}$ period using a custom written sequencer script. Identical measurements were repeated every $30 \mathrm{~s}$ in the all-out sprint.

\section{Data Analyses}

242 Data were analysed using a custom written script developed in Spike2 software (CED, 243 Cambridge, UK). For part A, mean torque for each $3 \mathrm{~s}$ contraction during the 60 MVC protocol was determined as the mean value over a 1-s period which approximated the plateau level of the highest torque (i.e. 500 ms before and after the peak torque). The pTw was calculated as the peak torque achieved following the single pulse delivered 1-s post-MVC.

247 The twitch torque superimposed onto the peak force production of the MVC (sTw) was 
calculated as the increment in torque immediately following the pulse during MVCs. The end-test torque (i.e. critical torque, CT) during the 60 MVC test was defined as the mean of the last 12 contractions (i.e., the last 60 s; Burnley, 2009; Morgan et al. 2018a). The torqueimpulse was calculated as the area under the torque-time curve by accumulating the time integral of each MVC (3 s).

Voluntary activation (VA, \%) was calculated using the interpolated twitch method from peripheral nerve stimulation (Merton, 1954; Goodall et al. 2010). Specifically, the increment in torque evoked during the MVCs was expressed as a fraction of the amplitude of the potentiated twitch produced with the same stimulus in the relaxed muscle post-MVC. The level of voluntary drive was then quantified as a percentage: [1 - evoked torque (superimposed on voluntary torque, sTw)/ (mean control evoked response, pTw) × 100] (i.e.,

Allen at al. 1998). The changes in voluntary torque and pTw, were used to assess global fatigue and peripheral fatigue, respectively with VA, $\mathrm{M}_{\text {Max }}$ and $E M G_{R M S}$ used to assess central fatigue. The maximal EMG was taken from the first MVC during the $60 \mathrm{MVC}$ task and compared to the last MVC at task end. The neuromuscular parameters extracted from the three sets of maximal contractions completed post-exercise were tested for statistical differences between sets of contractions and then compared to the first set of MVCs completed pre-exercise (Froyd et al. 2013; Pageaux et al. 2015; Doyle-Baker et al. 2017). Neuromuscular function was also measured for each of the stimulated contractions during the exercise and normalised to the corresponding pre-exercise values at 100\% MVC. defined as the mean of the last $30 \mathrm{~s}$ (Vanhatalo et al. 2007; 2008). The $\mathrm{W}^{\prime}$ was calculated as 272 the area above the CP from the power-time curve. Power output was recorded second-by- 
second, and the peak power was determined as the highest 1-s value. The changes in power output, $\mathrm{M}_{\mathrm{Max}}$ and $\mathrm{EMG}$ RMS, were used to quantify neuromuscular fatigue development and changes in muscle activation. Peak $\dot{\mathrm{VO}}_{2}$ was determined as the highest value recorded in a 15-s interval. The achievement of $\dot{\mathrm{V}} \mathrm{O}_{2 \text { peak }}$ was an essential criterion for a valid 3-min test. All neuromuscular parameters and torque were averaged across the protocol using 30-s bin averages.

\section{Statistics}

Paired-samples $t$-tests were used to compare the mean torque, total work done, CT, $\mathrm{W}^{\prime}$ and pTw between IBP and PL in part A. In addition, paired-samples t-tests were used to compare the total work done, $\mathrm{CP}, \mathrm{W}^{\prime}$ and cardiorespiratory responses between IBP and PL obtained from the 3-minute maximal cycling test. For the 60 MVC test, the profiles of VA and Mwave amplitude were analysed using two-way ANOVAs with repeated measures (using 12 contraction averages; i.e. 6 time points). In addition, a two-way ANOVA with repeated measures (condition $\times$ work rate) was used to assess differences in end exercise $\mathrm{V}_{2}$ and the profiles of EMG and M-wave amplitude. Normalised EMGRMS were analysed using two-way ANOVAs with repeated measures using 30-s means (i.e. 10 time points). Where sphericity was violated, Greenhouse-Geisser correction factor was used. For all tests, results were considered statistically significant when $P<0.05$. Data are presented as means \pm SD unless otherwise indicated. All statistical analyses were conducted using IBM SPSS Statistics version 23.

\section{RESULTS}


The mean MVC torque achieved prior to the 60 MVC protocol was $232 \pm 47$ and $230 \pm$ 55 N.m for PL and IBU, respectively. VA of the knee extensors achieved during the preliminary MVCs was $88 \pm 7$ and $89 \pm 6 \%$ for PL and IBU, respectively. Baseline MVC and VA were not different between conditions $(\mathrm{P}>0.05)$. The profile for mean torque during each contraction across all participants for the $60 \mathrm{MVC}$ protocol is illustrated in figure 2a. During the PL trial, torque declined from a peak of $99 \pm 3 \%$ MVC (relative to pre-exercise MVC) during the first contraction to $40 \pm 15 \%$ MVC during the last 12 contractions $(P<0.01$, Table 1, figure 2a). During the IBP trial torque declined from a peak of $99 \pm 3 \%$ to $41 \pm 16 \%$ MVC. The mean torque (relative to pre-exercise MVC) achieved across the 60 MVCs not different with IBP $(60 \pm 13 \%, 87.1 \pm 22.2$ N.m,) compared to PL (58 \pm 14\%, $83.8 \pm 22.7$ N.m, $P=0.39$ ). There was no difference in CT (PL: $40 \pm 15 \%$ vs. IBP: $41 \pm 16 \% . P=0.74$ ), $\mathrm{W}^{\prime}$ (PL: $6.97 \pm 2.43$ vs. IBP: $7.23 \pm 3.57$ N.m.s; $P=0.69$ ) or total impulse, the surrogate measure of total work done (PL: 22,055 \pm 3,885 vs. IBP: 22,919 \pm 3,394 N.m.s; $P=0.26$ ) between the PL and IBP conditions.

\section{Part A (Neuromuscular Function)}

Alongside the decline in voluntary force (figure 2a), pTw (figure 3a), VA (figure 3b),

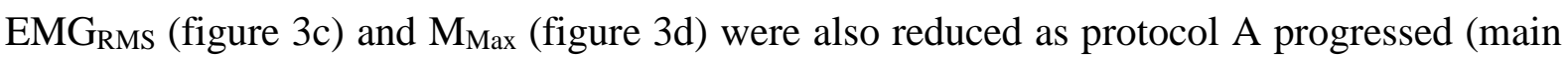
effect of time, all $P<0.01)$. There were no differences between PL and IBP in pTw, VA, EMG amplitude or M-wave amplitude at any time point (all $P>0.05$ ). pTw declined from 63 \pm 14 to $30 \pm 21 \mathrm{~N} \cdot \mathrm{m}$ and from $68 \pm 17$ to $31 \pm 23 \mathrm{~N} \cdot \mathrm{m}$, VA declined from $89 \pm 8$ to $59 \pm 19 \%$ and from $88 \pm 7$ to $60 \pm 18 \%$, EMG declined from $99 \pm 4$ to $59 \pm 17 \%$ and $100 \pm 2$ to $64 \pm$ 20\% (from first 6 to last 6 contractions) and M-wave amplitude declined from $100 \pm 1$ to $96 \pm$ $14 \%$ and $100 \pm 1$ to $95 \pm 12 \%$ in the PL and IBP conditions, respectively $(P<0.05)$, with no differences between PL and IPB for any of these variables $(P>0.05)$. 
Part B (3-min cycling test)

324

The mean power output profile for all participants during the 3-min all-out cycling test is shown in figure 2b for the PL and IBP conditions. During the PL trial, power output declined from $820 \pm 139 \mathrm{~W}$ to $288 \pm 31 \mathrm{~W}$ during the last $30 \mathrm{~s}$ of the 3 -min test $(P<0.01$; table 1 , figure 2b). During the IBP trial, power output declined from $816 \pm 131 \mathrm{~W}$ to $292 \pm 28 \mathrm{~W}$ during the last $30 \mathrm{~s}$ of the 3-min test. There were no differences in CP (PL: $288 \pm 31$ vs. IBP: $292 \pm 28 \mathrm{~W}, P=0.11$ ), total work done (PL: $65.4 \pm 6.4$ vs. IBP: $65.9 \pm 5.9 \mathrm{~kJ}, P=0.11$ ) or $\mathrm{W}^{\prime}$ (PL: $13.6 \pm 2.4$ vs. IBP: $13.7 \pm 2.8 \mathrm{~kJ}, P=0.84$ ) between conditions.

Part B (Neuromuscular Function)

Similar to the 60 MVC protocol, alongside the decline in power output, there was a progressive decline in M-wave ( $P<0.01$, figure $4 \mathrm{~b})$ and EMG amplitudes $(P<0.01$, figure $4 a)$.

The profiles of the M-wave and EMG amplitudes were not altered following IBP ingestion at any time point (both $P>0.05$ ). Using 30 s mean values, EMG decreased from $94 \pm 4$ to $54 \pm$ $17 \%$ and from $96 \pm 6$ to $57 \pm 14 \%$ (figure 4b) in PL and IBP, respectively.

\section{DISCUSSION}

Contrary to our experimental hypotheses, the principal findings of this study were that acute ingestion of $400 \mathrm{mg}$ of IBP in a 'fresh' state had no effect on fatigue development or neuromuscular function during either a 5-min single-leg intermittent MVC test or a 3-min maximal cycling test. Across both protocols, power output and torque declined, and neuromuscular fatigue was evident in both the IBP and PL conditions. However, there were no differences in CT or CP, total work done or neuromuscular fatigue markers between the 
IBP and PL conditions. These findings do not support the acute ingestion of IBP as a strategy

to blunt fatigue development during exercise in healthy, recreationally active adults.

\section{Effects of acute IBP ingestion on exercise performance}

350 In contrast with our previous findings of improved CT and CP following the acute ingestion of ACT (Morgan et al. 2018a; Morgan et al. 2018b), these variables were not improved in the current study following the acute ingestion of IBP. However, our findings in the current study are in line with some previous observations that IBP ingestion does not improve performance during whole body exercise in humans (e.g., Cleak, \& Eston. 1992; Da Silva et al. 2015;

Nosaka \& Clarkson, 1996; Tokmakdis et al. 2003). Previous studies that have reported improved exercise performance following IBP administration have typically assessed exercise performance following muscle damage. Nonetheless, while there is some evidence to suggest that the increases in muscle soreness, pain, damage and contractile dysfunction after contraction-induced muscle damage can be attenuated following NSAID administration (Ebbeling \& Clarkson, 1989; Hasson et al. 1993; Pizza et al. 1999; Tokmakdis et al. 2003), there is also evidence that NSAID administration does not impact these variables after muscle damage is induced (Da Silva et al. 2015; Tokmakdis et al. 2003). Taken together, these observations suggest that the ergogenic potential of administering IBP, a purported analgesic and anti-inflammatory agent (Friden \& Lieber, 1992), is limited without prior induction of muscle damage, and even when muscle damage is induced, and inflammation and pain sensation are correspondingly increased, the effect of IBP ingestion on exercise performance is equivocal.

One explanation for the lack of performance improvement following IBP ingestion in our 
the maximal protocols employed in response to a potential modulation of pain. For example, Mauger et al. (2010) reported that pain`sensation was reduced (i.e., a greater power output was possible for the same pain sensation) and cycling time trial performance was enhanced following ingestion of ACT. Similarly, Gonglach et al. (2016) reported that manipulating pain perception via caffeine ingestion lead to higher power outputs when participants were asked to pace their effort based upon pain perception. Therefore, we cannot exclude the possibility that IBP consumption could be ergogenic in situations wherein pacing strategy is self-selected such as during longer duration endurance exercise.

Another possible explanation for the absence of an ergogenic effect of IBP in our study is that inhibiting cyclooxygenase may induce secondary effects that may limit the cardiopulmonary response to exercise (Takayama et al. 2002) including a reduction in exercise-induced hyperaemia (Bradford et al. 2007; Scharage et al. 2004). If skeletal muscle perfusion was impaired following IBP ingestion as a function of cyclooxygenase inhibition (Albert \& Gernaat, 1984), this could have negated any potential ergogenic effects following IBP ingestion. However, given that performance was not enhanced during either smaller (singleleg exercise) or larger (double leg cycling) muscle mass exercise following acute IBP ingestion in the current study, and that skeletal muscle perfusion is less likely to be a limiting factor for performance in a single leg model (Joyner \& Casey, 2015), this seems unlikely.

\section{Effects of acute IBP ingestion on neuromuscular function}

The ingestion of $400 \mathrm{mg}$ of IBP was not associated with attenuation of neuromuscular fatigue development as estimated with peripheral muscle excitability (part A and B), voluntary activation (part A), potentiated twitch (part A) or EMG amplitude (part A and B), consistent with the lack of change in exercise performance. We previously investigated the influence of 
acute ingestion of $1 \mathrm{~g}$ of ACT on neuromuscular function during exercise and reported that, whilst EMG declined across the 3-min cycling protocol in both conditions, EMG declined to a lesser extent in the ACT ( 72 \%) compared to the PL ( 54 \%) condition (Morgan et al. 2018b). The magnitude of this effect was remarkably similar to the results we obtained during a 60 MVC protocol completed in a single leg exercise model (ACT: 87\% vs. PL: 59 \%, Morgan et al. 2018a). This observation suggests that improved maintenance of muscle activation contributed to the ergogenic effect of ACT (Morgan et al. 2018a). It is of interest, therefore, that similar effects were not evident following IBP ingestion. We are not aware of any research to suggest that ACT acts as a more potent stimulus to reduce pain sensation or alter muscle activation. However, it is possible that a combination of the higher ACT dose administered (1000 mg vs 400 mg for IBU), ACT's potential as an antipyretic (Foster et al. 2014) and differences in pharmacokinetics (Anderson, 2008; Albert \& Gernaat, 1984) may have contributed to the disparate effects of IBP in our current study and ACT in our previous studies (Morgan et al. 2018a; Morgan et al. 2018b) on exercise performance and neuromuscular fatigue development. It is also pertinent to note that ACT ingestion has been reported to increase corticospinal excitability at rest, a factor which may contribute to its ergogenic potential (Mauger \& Hopker, 2013).

\section{Experimental considerations}

Whilst our study contributes to a further understanding of the effect of IBP on exerciseinduced fatigue and some of its underlying mechanisms, there are some limitations that require consideration. Firstly, it is acknowledged that we did not measure pain sensation or biomarkers of inflammation. Pain was not assessed due to the protocols requiring the completion of maximal exercise. Asking participants to rate pain sensation may have compromised their ability to focus on the exercise task and to provide a true maximal effort. 
421 The effect of IBP on exercise performance when pacing is permitted, and the individual can

422 adjust their pacing strategy in response to potential differences in pain sensation, warrants

423 further investigation. In addition, the extent to which prostaglandin synthesis during

424 endurance exercise plays a role in pain perception in the absence of muscle damage requires

425 further consideration. It is also important to point out that individuals intending to ingest IBP

426 need to consider its potential side effects and be aware that it could impair the adaptive

427 response to exercise (Schoenfield, 2012) and aspects of the beneficial remodelling of skeletal

428 muscle to exercise training (Mikkelsen, 2009). Therefore, individuals wishing to explore the

429 use of pain relievers to enhance exercise performance should do so infrequently, with

430 caution, and at the recommended therapeutic doses.

431

432 Conclusion

433 Acute ingestion of IBP did not attenuate the decline in neuromuscular function or improve

434 CT or CP during a 60 MVC protocol of the knee extensors or a 3-min maximal cycling test.

435 Therefore, our results indicate that IBP ingestion does not attenuate neuromuscular fatigue

436 development, during either single-limb or whole-body cycling exercise, and do not support

437 IBP ingestion as an ergogenic aid. 


\section{Conflict of interest}

439 The author declares that there is no conflict of interest regarding the publication of this 440 article.

441

442 Author contribution

443 P.T. Morgan, A. Vanhatalo, A.M. Jones and S.J. Bailey conceived and designed the research.

444 P.T. Morgan conducted all experiments. J.L. Bowtell provided assistance with pilot testing 445 prior to experimental data collection as well supporting data analysis. P.T. Morgan wrote the 446 manuscript. J.L. Bowtell, A. Vanhatalo, A.M. Jones and S.J. Bailey helped supervise the 447 project throughout. All authors contributed to the interpretation of results and read, edited and 448 approved the manuscript. 
Albert, K.S., and Gernaat, C.M. 1984. Pharmacokinetics of Ibuprofen. American Journal of Medicine. 77: 40-46.

452

Alaranta, A., Alaranta, H., and Helenius, I. 2008. Use of prescription drugs in athletes. Sports

Medicine. 38: 449-463.

455

456

Allen, G.M., McKenzie, D.K., and Gandevia, S.C. 1998. Twitch interpolation of the elbow

flexor muscles at high forces. Muscle Nerve. 21(3): 318-28.

458

459

460

Amann, M., and Calbet, J.A.L. 2008. Convective oxygen transport and fatigue. Journal of Applied Physiology. 104: 861-870.

461

462 performance in humans. Journal of Physiology. 589: 5299-5309.

465

466

467

468 development in humans. Journal of Physiology. 587: 271-283.

469

470

Amann, M., Venturelli, M., Ives, S.J., McDaniel, J., Layec, G., Rossman, M.J., and

471

Richardson, R.S. 2013. Peripheral fatigue limits endurance exercise via a sensory feedback-

472 mediated reduction in spinal motoneuronal output. Journal of Applied Physiology. 115: 55473 364. 
Anderson, B.J. 2008. Paracetamol (Acetaminophen): mechanisms of action. Paediatric

Anaesthesia. 18: 915-921.

477

Armstrong, R.B. 1984. Mechanisms of exercise-induced delayed onset muscular soreness.

Medicine \& Science in Sports \& Exercise. 16: 529-538.

480

481

Black, M.I., Durant, J., Jones, A.M., and Vanhatalo, A. 2014. Critical power derived from a 482 3-min all-out test predicts 16.1-km road time-trial performance. European Journal of Sport

Science. 14: 217-223.

484

Black, C.D., and O’Connor, P.J. 2008. Acute effects of dietary ginger on quadriceps muscle 486 pain during moderate-intensity cycling exercise. International Journal of Sport Nutrition and Exercise Metabolism. 18: 653-664.

Blain, G.M., Mangum, T.S., Sidhu, S.K., Weavil, J.C., Hureau, T.J., Jessop, J.E., Bledsoe, 490 A.D., Richardson, R.S., and Amann, M. 2016. Group III/IV muscle afferents limit the 491 intramuscular metabolic perturbation during whole body exercise in humans. Journal of Physiology. 594: 5303-5315.

493

Bradford, C.D., Cotter, J.D., Thorburn, M.S., Walker, R.J., and Gerrard, D.F. 2007. Exercise can be pyrogenic in humans. American Journal of Physiology. Regulatory, Integrative and Comparative Physiology 292: R143-9.

497 
498

499

500

501

502

503

504

505

506

507

508

509

510

511

512

513

514

515

516

517

518

519

520

521

522

Burnley, M. 2009. Estimation of critical torque using intermittent isometric maximal

voluntary contractions of the quadriceps in humans. Journal of Applied Physiology. 106: 975-983.

Cleak, M.J., and Eston, R.G. 1992. Muscle soreness, swelling, stiffness and strength loss after intense eccentric exercise. British Journal of Sports Medicine. 26: 267-272.

Cook, D.B., O’Connor, P.J., Eubanks, S.A., Smith, J.C., and Lee, M. 1997. Naturally

occurring muscle pain during exercise: assessment and experimental evidence. Medicine and Science in Sports and Exercise. 29: 999-1012.

Corrigan, B., and Kazlauskas, R. 2003. Medication use in athletes selected for doping control at the Sydney Olympics (2000). Clinical Journal of Sport Medicine. 13: 33-40.

Da Silva, E., Pinto, R.S., Cadore, E.L., and Kruel, L.F. 2015. Nonsteroidal anti-inflammatory drug use and endurance during running in male long-distance runners. Journal of Athletic Training. 50: 295-302.

Donnelly, A.E., Maughan, R.J., and Whiting, P.H. 1990. Effects of ibuprofen on exerciseinduced muscle soreness and indices of muscle damage. British Journal of Sports Medicine. 24: 191-195.

Doyle-Baker, D., Temesi, J., Medysky, M.E., Holash, R.J., and Millet, G.Y. 2017. An Innovative Ergometer to Measure Neuromuscular Fatigue Immediately after Cycling. Medicine \& Science in Sports \& Exercise. 50(2): 375-387. 
524 Ebbeling, C.B., and Clarkson, P.M. 1989. Exercise-Induced Muscle Damage and Adaptation. 525 Sports Medicine. 7: 207-234.

526

527 Foster, J., Taylor, L., Chrismas, B.C., Watkins, S.L., and Mauger, A.R. (2014). The influence 528 of acetaminophen on repeated sprint cycling performance. European Journal of Applied 529 Physiology. 114: 41-48.

530

531 Fridén, J., and Lieber, R.L. 1992. Structural and mechanical basis of exercise-induced muscle 532 injury. Medicine and Science in Sports and Exercise. 24: 521-530.

533

534 Froyd, C., Millet, G.Y., and Noakes, T.D. 2013. The development of peripheral fatigue and 535 short-term recovery during self-paced high-intensity exercise. Journal of Physiology. 591: 536 1339-1346.

537

538 García-Martín, E., Martínez, C., Tabarés, B., Frías, J., and Agúndez, J.A.G. 2004. Interindividual variability in ibuprofen pharmacokinetics is related to interaction of cytochrome P450 2C8 and 2C9 amino acid polymorphisms. Clinical Pharmacology and

541 Therapeutics. 76: 119-127.

542

543 Goodall, S., Ross, E.Z., and Romer, L.M. 2010. Effect of graded hypoxia on supraspinal 544 contributions to fatigue with unilateral knee-extensor contractions. Journal of Applied 545 Physiology. 109: 1842-1851. 
Gonglach, A.R., Ade, C.J., Bemben, M.G., Larson, R.D., and Black, C.D. 2016. Muscle pain as a regulator of cycling intensity: Effect of caffeine ingestion. Medicine and Science in Sports and Exercise. 48: 287-296.

550

Gorski, T., Lusa Cadore, E., Santana Pinto, S., Marczwski Da Silva, E., Silva Correa, C.,

552 Gabe Beltrami, F., and Martins Kruel, L.F. 2011. Use of NSAIDs in triathletes: Prevalence, level of awareness and reasons for use. British Journal of Sports Medicine. 45: 85-90.

554

Grossman, J.M., Arnold, B.L., Perrin, D.H., and Kahler, D.M. 1995. Effect of Ibuprofen Use on Delayed Onset Muscle Soreness of the Elbow Flexors. Journal of Sport Rehabilitation. 4: 253-263.

558

559

Hasson, S.M., Daniels, J.C., Divine, J.G., Niebuhr, B.R., Richmond, S., Stein, P.G., and

Williams, J.H. 1993. Effect of ibuprofen use on muscle soreness, damage, and performance: a preliminary investigation. Medicine \& Science in Sports \& Exercise. 25: 9-17.

562

Huang, S.H., Johnson, K., and Pipe, A.L. 2006. The use of dietary supplements and medications by Canadian athletes at the Atlanta and Sydney olympic games. Clinical Journal of Sport Medicine. 16: 27-33. R.S., and Kohrt, W.M. 2015. Timing of ibuprofen use and musculoskeletal adaptations to exercise training in older adults. Bone Reports. 1: 1-8. 
Janssen, G.M.E., and Venema, J.F. 1985. Ibuprofen: Plasma Concentrations in Man. Journal of International Medical Research. 13: 68-73.

573

574 Joyner, M.J., and Casey, D.P. 2015. Regulation of Increased Blood Flow (Hyperemia) to 575 Muscles During Exercise: A Hierarchy of Competing Physiological Needs. Physiological 576 Reviews. 95: 549-601.

577

Leadbetter, W.B. 1995. Anti-inflammatory therapy in sports injury. The role of nonsteroidal 579 drugs and corticosteroid injection. Clinics in Sports Medicine. 14: 353-410.

580

581

Mauger, A.R., and Hopker, J.G. 2013 The effect of acetaminophen ingestion on cortico582 spinal excitability. Canadian Journal of Physiology and Pharmacology. 91: 187-189.

583

Mauger, A.R., Jones, A.M., and Williams, C.A. 2010. Influence of acetaminophen on performance during time trial cycling. Journal of Applied Physiology. 108: 98-104.

586

587

588

Mauger, A.R., Taylor, L., Harding, C., Wright, B., Foster, J., and Castle, P.C. 2014. Acute acetaminophen (paracetamol) ingestion improves time to exhaustion during exercise in the heat. Experimental Physiology. 99: 164-171.

590

591

McCord, J.L., and Kaufman, M.P. 2010. Reflex Autonomic Responses Evoked by Group III and IV Muscle Afferents. In: L. Kruger \& A.R. Light (eds). Translational Pain Research: From Mouse to Man, Chapter 12, pp 283-300. CRC Press/Taylor \& Francis, Boca Raton, FL. 
597

598

599

600

601

602

603

604

605

606

607

608

609

610

611

612

613

614

615

616

617

618

619

Merton, P.A. 1954. Voluntary strength and fatigue. The Journal of Physiology. 123: 553564.

Mikkelsen, U.R., Langberg, H., Helmark, I.C., Skovgaard, D., Andersen, L.L., Kjaer, M., and Mackey, a L. 2009. Local NSAID infusion inhibits satellite cell proliferation in human skeletal muscle after eccentric exercise. Journal of Applied Physiology. 107: 1600-1611.

Morgan, P.T., Bowtell, J.L., Vanhatalo, A., Jones, A.M., and Bailey, S.J. 2018a. Acute acetaminophen ingestion improves performance and muscle activation during maximal intermittent knee extensor exercise. European Journal of Applied Physiology. 118: 595-605.

Morgan, P.T., Bowtell, J.L., Vanhatalo, A., Jones, A.M., and Bailey, S.J. 2018b.

Acetaminophen improves muscle activation and performance during 3-min all-out cycling. Applied Physiology, Nutrition, and Metabolism (under review).

Nosaka, K., and Clarkson, P.M. 1996. Changes in indicators of inflammation after eccentric exercise of the elbow flexors. Medicine and Science in Sports and Exercise. 28: 953-961.

Novak, J., and Wennmalm, A. 1979. Effect of exercise on human arterial regional venous plasma concentrations of prostaglandin E. Prostaglandins and Medicine.1: 489-497.

O'Connor, P.J., and Cook, D.B. 1999. Exercise and pain: the neurobiology, measurement, and laboratory study of pain in relation to exercise in humans. Exercise and Sport Sciences Reviews. 27: 119-66. 
Pageaux, B., Angius, L., Hopker, J.G., Lepers, R., and Marcora, S.M. 2015. Central alterations of neuromuscular function and feedback from group III-IV muscle afferents following exhaustive high-intensity one-leg dynamic exercise. American Journal of Physiology - Regulatory, Integrative and Comparative Physiology. 308: R1008-R1020.

Pizza, F., Calende, D., Stockard, A., Baylies, H., and Beighle, A. 1999. Anti-Inflammatory Doses of Ibuprofen: Effect on Neutrophils and Exercise-Induced Muscle Injury. International Journal of Sports Medicine. 20: 98-102.

Pollak, K.A., Swenson, J.D., Vanhaitsma, T.A., Hughen, R.W., Jo, D., White, A.T., Light, K.C., Schweinhardt, P., Amann, M., and Light, A.R. 2014. Exogenously applied muscle metabolites synergistically evoke sensations of muscle fatigue and pain in human subjects. Experimental Physiology. 99: 368-380.

Schrage, W.G., Joyner, M.J., and Dinenno, F.A. 2004. Local inhibition of nitric oxide and prostaglandins independently reduces forearm exercise hyperaernia in humans. Journal of Physiology. 557: 599-611.

Schoenfeld, B.J. 2012. The use of nonsteroidal anti-inflammatory drugs for exercise-induced muscle damage: Implications for skeletal muscle development. Sports Medicine. 42: 10171028.

Tokmakidis, S.P., Kokkinidis, E.A., Smilios, I., and Douda, H. 2003. The effects of ibuprofen on delayed muscle soreness and muscular performance after eccentric exercise. Journal of Strength and Conditioning Research. 17: 53-59. 
646 Trappe, T.A., Fluckey, J.D., White, F., Lambert, C.P., and Evans, W.J. 2001. Skeletal muscle 647 PGF2 $\alpha$ and PGE2 in response to eccentric resistance exercise: Influence of ibuprofen and 648 acetaminophen. Journal of Clinical Endocrinology and Metabolism. 86: 5067-5070.

650 Vane, J.R. 2000. Aspirin and other anti-inflammatory drugs. Thorax. 231(25): 232-235.

651

652 Vane, J.R., and Botting, R.M. 2003. The mechanism of action of aspirin. In Thrombosis 653 Research, (Elsevier Ltd). 255-258.

654

655

Vanhatalo, A., Doust, J.H., and Burnley, M. 2007. Determination of critical power using a 3656 min all-out cycling test. Medicine and Science in Sports and Exercise. 39: 548-555.

657

658

Vanhatalo, A., Doust, J. H., and Burnley, M. 2008. A 3-min all-out cycling test is sensitive to a change in critical power. Medicine and Science in Sports and Exercise. 40(9): 1693-1699.

660

661

VanHeest, J., Stoppani, J., TP, S., Collins, V., Roti, M., Anderson, J., ... CM, M. 2002.

662

Effects of ibuprofen and Vicoprofen on physical performance after exercise-induced muscle

663 damage. Journal of Sport Rehabilitation. 11: 224-234.

664

665 Vinikka, L., Vuori, J., and Ylikorkala, O. 1984. Lipid peroxidases, prostacyclin, and 666 thromboxane A2 in runners during acute exercise. Medicine \& Science in Sports \& Exercise.

667 16: $275-277$. 
Table 1: Performance and neuromuscular function parameters of the $60 \mathrm{MVC}$ (protocol A) and 3-min all-out cycling (protocol B) tests following placebo and ibuprofen ingestion.

\begin{tabular}{lcc}
\hline & Placebo (PL) & Ibuprofen (IBU) \\
\hline Protocol A & & \\
Performance & $232 \pm 47$ & $230 \pm 55$ \\
Peak MVC (N.m) & $58 \pm 14$ & $60 \pm 13$ \\
Mean torque (\% MVC) & $22055 \pm 3885$ & $22919 \pm 3394$ \\
Total Impulse (N.m.s) & $40 \pm 15$ & $41 \pm 16$ \\
Critical torque, CT (\% MVC) & $6971 \pm 2432$ & $7231 \pm 3570$ \\
W' (N.m.s $^{-1}$ ) & & \\
& & \\
Neuromuscular function & $44 \pm 27$ & $43 \pm 25$ \\
Change in pTw (N.m) & $30 \pm 21$ & $31 \pm 23$ \\
$\quad$ End-exercise pTw (N.m) & $59 \pm 19$ & $60 \pm 18$ \\
End-exercise VA (\%) & $96 \pm 14$ & $95 \pm 12$ \\
End-exercise M-wave amplitude (\%) & $59 \pm 17$ & $64 \pm 20$ \\
End-exercise EMG amplitude (\%) & & $816 \pm 131$ \\
\hline Protocol B & & $65.9 \pm 5.9$ \\
Performance & $820 \pm 139$ & $292 \pm 28$ \\
Peak power output (W) & $65.4 \pm 6.4$ & $13.7 \pm 2.8$ \\
Total Work Done (kJ) & $288 \pm 31$ & \\
Critical power, CP (W) & $13.6 \pm 2.4$ & $83 \pm 18$ \\
W' (kJ) & & $57 \pm 14$ \\
Neuromuscular function & $84 \pm 21$ & \\
End-exercise M-wave amplitude (\%) & $54 \pm 17$ & \\
End-exercise EMG amplitude (\%) & & \\
& & \\
\hline
\end{tabular}

$\mathrm{MVC}$, maximal voluntary contraction; $\mathrm{CT}$, critical torque measured in the last 6 contractions; $\mathrm{CP}$, critical power measured in the last $30 \mathrm{~s}$ of the 3-minute maximal cycling test; pTw, potentiated twitch force; VA, voluntary activation measured using the interpolated twitch method technique; EMG, electromyography; N.m, newton metres; N.m.s-1, newton metres per second; ms, milliseconds. 


\section{Figure captions}

670

671

672

673

674

675

676

677

678

679

680

681

682

683

684

685

686

687

688

689

690

691

692

Figure 1

Schematic of the procedures used prior to (panel a), during (panel b) and within $10 \mathrm{~s}$ following (panel c) the 60 maximal isometric voluntary contraction (MVC) protocol. $10 \mathrm{~s}$ separated each single pulse stimulation administered at rest (small dashed arrows). A, C $45 \mathrm{~s}$ rest period separated maximal efforts (MVCs). Single pulse stimuli were administered during peak force production of MVCs (large solid arrow) and immediately $(<1-$ s) post MVCs (small grey arrows). B: 60 MVC protocol of the knee extensors. The figure presents a period of $30 \mathrm{~s}$ which is repeated sequentially for $5 \mathrm{~min}$. Each MVC was held for $3 \mathrm{~s}$ and interspersed by a 2 s passive recovery period. Every $6^{\text {th }}$ MVC was accompanied by single pulse stimuli administered during peak force production (large solid arrow) and immediately following (>1 s) post MVCs (small grey arrows). This cycle was repeated 10 times such that the protocol spanned 5 minutes requiring the completion of 60 MVCs. Surface electromyography (EMG) was measured throughout.

\section{Figure 2}

The torque profile during the 60 maximal contractions for placebo (PL, clear circles) and ibuprofen (IBU, filled circles) trials in protocol (a) is demonstrated in panel A. The torque during all contractions was normalized to a control maximal voluntary contraction (MVC) performed before the test commenced. Note that torque falls over the first $150 \mathrm{~s}$ before reaching stable values between 240 and 300 s (the end-test torque; last 12 MVCs). Panel B illustrates the mean \pm SE power output profile during the 3-min maximal cycling protocol for placebo (clear circles) and ibuprofen (filled circles) trials. Note that power output falls over the first $\sim 120$-150 s before reaching stable values (the end-test power output; i.e. CP). 
694 Figure 3

695 Mean \pm SE potentiated twitch (A), voluntary activation (B), and EMG amplitude (C) and M696 wave amplitude (D) responses during the 60 MVC test for placebo (clear circles) and 697 ibuprofen (filled circles) trials for protocol a.

698

699 Figure 4

700 Mean \pm SE M-wave amplitude (A) and EMG amplitude (B) responses during the 3-minute 701 maximal cycling exercise for placebo (clear circles) and ibuprofen (filled circles) trials for 702 protocol b. 